\title{
Explorando Longo Período de Interação entre Sistema Imunológico e HIV ${ }^{1}$
}

A.M. MALAQUIAS 2 , H.M. YANG ${ }^{3}$, Departamento de Matemática Aplicada, Instituto de Matemática, Estatística e Computação Científica, UNICAMP e LabEpifisma, Cx.P. 6065, 13083-859 Campinas, SP, Brasil.

Resumo. O Vírus da Imunodeficiência Humana (HIV), infectando células T$\mathrm{CD} 4^{+}$, consegue comprometer gravemente o funcionamento do Sistema Imunológico, resultando em AIDS. Formulamos e analisamos três modelos matemáticos para este processo de infecção. Foi possível encontrar um limiar para a permanência do vírus na corrente sanguínea e evidenciar a necessidade da apoptose de células ativadas, após a eliminação do antígeno. Através da introdução de um termo de exaustão cumulativa (resultado da constante tentativa do sistema imunológico responder à infecção pelo HIV) também reproduzimos a diminuição do número de células T-CD4 ${ }^{+}$ao longo dos anos.

\section{Introdução}

O corpo humano é formado por um complexo sistema de defesa, o Sistema Imunológico (SI) [1] [8], que é capaz de identificar e responder de modo específico a cada elemento estranho ao organismo. Apesar de sua eficiência, o Vírus da Imunodeficiência Humana (HIV) é um vírus que pode escapar de suas defesas e deixá-lo bastante comprometido [14].

Com o avanço do conhecimento sobre HIV [2] [6], muitos estudos foram realizados na tentativa de contê-lo. Atualmente, grande parte dos esforços provém de terapias antiretrovirais (ARV's) e com esforço de desenvolver uma vacina. Embora existam avanços neste sentido, para se propor estratégias de controle à propagação da infeç̧ão, é necessário compreender a interação entre as células do SI e o HIV.

Modelos matemáticos têm auxiliado na compreensão e no estudo da dinâmica do HIV [3] [4] [10] [11] [12] [13]. Este trabalho difere dos citados pela inclusão de termos ainda não modelados, e por reproduzir o progresso natural da infecção pelo HIV até a fase avançada, que pode ser caracterizada como AIDS.

O SI é formado por um complexo de proteínas e células (e.g., macrófagos, linfócitos e Natural Killer) que, em conjunto, agem para manter nosso organismo livre de infecções. Dentre estas células destacamos os linfócitos, por responderem de

\footnotetext{
${ }^{1}$ Apoio financeiro da FAPESP - Projeto Temático

${ }^{2}$ malaquias@ime.unicamp.br.

${ }^{3}$ hyunyang@ime.unicamp.br. Bolsista CNPq.
} 
maneira específica a um determinado antígeno (vírus) e estimularem a ação de outras células. Os linfócitos, assim como todas as células sanguíneas, são produzidos na medula óssea. Após completarem todos seus estágios de amadurecimento, na medula óssea (linfócitos B) ou no timo (linfócitos T), entram na circulação e se espalham pelo corpo, passando a ser chamados de linfócitos virgens ou inativos.

Quando linfócitos inativos são expostos a antígenos que de alguma forma entram na corrente sanguínea (ferimentos nas mucosas, injeção por agulha, etc.), eles são ativados e se diferenciam. As células B são as responsáveis pela produção de anticorpos, proteínas que se ligam ao vírus deixando-os marcados para a eliminação por outras células, ou desencadeando processos que levam a eliminação dos mesmos. Quanto aos linfócitos T, são constituídos basicamente por duas classes: T-CD4 ${ }^{+}$ e T-CD8 ${ }^{+}$. A primeira é responsável por emitir estímulos para o crescimento e diferenciação dos linfócitos B e T, e ativar macrófagos pelas proteínas secretadas (citocinas), enquanto a segunda, pela destruição de células infectadas pelo vírus.

O resultado da infecção pelo HIV é caracterizado principalmente pela redução do número de células $\mathrm{T}_{-} \mathrm{CD} 4^{+}$que, após infectadas, podem produzir novas partículas virais, possibilitando assim a propagação da infecção. As T-CD4 ${ }^{+}$infectadas são mortas devido a ação do vírus, e a redução do número destas células compromete gravemente o SI, uma vez que desempenham um papel crucial na resposta específica ao antígeno. A contagem normal de T-CD $4^{+}$em um indivíduo adulto está entre 500 e 1600 células $/ \mathrm{mm}^{3}$, quando abaixo de 200 células $/ \mathrm{mm}^{3}$, tem-se um risco bastante elevado para doenças oportunistas.

A variação do número de T-CD4 ${ }^{+}$e da quantidade de vírus no plasma sanguíneo (carga viral), após a infecção pelo HIV, segue um curso característico. Inicialmente, enquanto o SI monta a defesa específica à infecção, ocorre uma elevação da carga viral. Posteriormente, por volta de 3 a 6 meses após a infecção, segue-se a fase assintomática que dura alguns anos. Ainda assim, contagens de T-CD4 ${ }^{+}$declinam gradualmente. Quando a concentração de T-CD4 ${ }^{+}$atinge valores por volta de 200 células $/ \mathrm{mm}^{3}$, o número de partículas virais torna a crescer, e podem ocorrer doenças oportunistas. Neste estágio, um indivíduo pode ser classificado como sintomático (AIDS, Síndrome da Imunodeficiência Adquirida).

\section{Modelo Base}

O processo de infecção foi estudado levando em consideração a concentração de partículas virais livres na corrente sanguínea e sua destruição, dada de maneira indireta, pela ação de anticorpos e células T-CD $8^{+}$. As variáveis $V(t), Y(t)$ e $Z(t)$ representam, respectivamente, as concentrações de vírus, linfócitos inativos (células $\mathrm{T}_{-\mathrm{CD}}{ }^{+}$que ainda não entraram em contato com o vírus) e linfócitos ativados (compostos pelas células B e T, estimuladas pelas T-CD4 ${ }^{+}$que iniciaram a resposta imunológica). Por simplicidade, embora as células B e T formem classes distintas, reunimos estas duas em $Z$, a qual chamaremos de população de células efetoras, dado que são elas que promoverão o ataque ao vírus.

O primeiro modelo segue das seguintes considerações: (i) as células inativas podem ser produzidas pela medula óssea a uma taxa $K$, podem morrer, $\mu_{Y} Y$, 
ser infectadas, $\alpha V Y$, ou ativadas para iniciar uma resposta imunológica, $\beta Y V$; (ii) a concentração viral pode aumentar devido a produção de vírus pelas células infectadas, $a(1-f) \alpha Y V$, e diminuir tanto por erros de replicação, $\mu_{V} V$, quanto pela ação de anticorpos, $\eta V Z$, e de células T-CD $8^{+}$, onde $0 \leq f \leq 1$ é um parâmetro associado à ação de células citotóxicas; e (iii) a população de células efetoras pode aumentar devido a ativação, $\beta Y V$, e diferenciação, $\gamma Z$, e, por outro lado, diminui pela mortalidade, $\mu_{Z} Z$. A partir destas considerações, temos o modelo descrito por

$$
\begin{aligned}
& \frac{d V}{d t}=a(1-f) \alpha Y V-\mu_{V} V-\eta V Z \\
& \frac{d Y}{d t}=K-\mu_{Y} Y-\alpha V Y-\beta V Y \\
& \frac{d Z}{d t}=\beta V Y-\mu_{Z} Z+\gamma Z .
\end{aligned}
$$

Quanto aos parâmetros, têm-se: $a$, quantidade de vírus liberada por célula T-CD4 ${ }^{+}$ infectada; $f$, ação das células T-CD8 ${ }^{+} ; \alpha$, taxa de infecção das células inativas devido ao contato com o vírus; $\mu_{V}$, taxa de inativação (replicação defeituosa, sair da circulação sanguínea, etc); $\eta$, taxa de eliminação de vírus devido a ação de anticorpos; $K$, taxa de reposição de células inativas pela medula óssea; $\mu_{Y}$, taxa de mortalidade das células inativas; $\beta$, taxa de ativação das células inativas; $\mu_{Z}$, taxa de mortalidade das células ativadas, e $\gamma$, taxa de diferenciação das células ativadas.

Representando por $T$, a dimensão de tempo, e $C$, de concentração, as dimensões das variáveis e taxas, que aparecem no modelo, são dadas por: $[V]=[Y]=[Z]=C$, $[\alpha]=[\beta]=[\eta]=C^{-1} T^{-1},[K]=C T^{-1}$ e $[\gamma]=\left[\mu_{V}\right]=\left[\mu_{Y}\right]=\left[\mu_{Z}\right]=T^{-1}$, sendo os parâmetros $a$ e f adimensionais. Faz-se a mudança de variáveis $V^{*}=V / Y_{h}$, $Y^{*}=Y / Y_{h}$ e $Z^{*}=Z / Y_{h}$, onde $Y_{h}=K / \mu_{Y}$, e para parâmetros, $\mu_{V}^{*}=\mu_{V} / A \alpha Y_{h}$, $\mu_{Y}{ }^{*}=\mu_{Y} / A \alpha Y_{h}, \alpha^{*}=1 / A, \beta^{*}=\beta / A \alpha, \eta^{*}=\eta / A \alpha, \mu_{Z}{ }^{*}=\mu_{Z} / A \alpha Y_{h}$ e $\gamma^{*}=$ $\gamma / A \alpha Y_{h}$, em que $A=a(1-f)$. Omitindo os asteriscos, o sistema (2.1) toma a forma adimensional

$$
\begin{aligned}
& \frac{d V}{d t}=Y V-\mu_{V} V-\eta V Z \\
& \frac{d Y}{d t}=\mu_{Y}-\mu_{Y} Y-\alpha V Y-\beta V Y \\
& \frac{d Z}{d t}=\beta V Y-\mu_{Z} Z+\gamma Z .
\end{aligned}
$$

Note que, agora, $Y, V$ e $Z$ representam a relação entre as variáveis antigas e a constante $Y_{h}=K / \mu_{Y}$, que representa a concentração de células T-CD4 ${ }^{+}$no estado de homeostase, situação em que o SI tenta manter um número constante de células.

Calcula-se os pontos de equilíbrio, ou seja, em (2.2) impomos $d V / d t=d Y / d t=$ $d Z / d t=0$. Para indicar que os valores de $V, Y$ e $Z$ estão no equilíbrio, não estão mais variando com o tempo, utilizamos as notações $\bar{V}, \bar{Y}$ e $\bar{Z}$. O sistema (2.2) possui dois pontos de equilíbrio. O trivial, estado em que não há vírus, e um não-trivial, em que as populações de vírus e linfócitos coexistem. O primeiro, $E_{0}=(\bar{V}, \bar{Y}, \bar{Z})=(0,1,0)$, é obtido de (2.2) impondo $V=0$, e resolvemos as equações resultantes, encontrando $Y$ e $Z$. O equilíbrio trivial corresponde ao caso 
em que indivíduos não entraram em contato com vírus, ou, em algumas situações, indivíduos que, para $t$ suficientemente grande, a resposta do SI consegue eliminar as partículas virais. O segundo ponto de equilíbrio, $E_{1}=(\bar{V}, \bar{Y}, \bar{Z})$, é dado por

$$
\left(\frac{\mu_{Y}\left(\mu_{Z}-\gamma\right)\left(1-\mu_{V}\right)}{\mu_{V}\left(\mu_{Z}-\gamma\right)(\alpha+\beta)+\eta \beta \mu_{Y}}, \quad \frac{\mu_{Y}}{\mu_{Y}+(\alpha+\beta) \bar{V}}, \frac{\beta \overline{V Y}}{\mu_{Z}-\gamma}\right) .
$$

Encontramos $E_{1}$ seguindo os mesmos passos como para $E_{0}$, mas com $V \neq 0$.

Para analisar a estabilidade local dos pontos de equilíbrio utilizamos técnicas de linearização. O ponto $E_{0}=(0,1,0)$ será Local e Assintoticamente Estável (L.A.E) se

$$
\mu_{V}>1 \text { e } \mu_{Z}>\gamma
$$

Isto pode ser verificado observando que $\lambda_{1}=1-\mu_{V}, \lambda_{2}=-\mu_{Y}$ e $\lambda_{3}=-\left(\mu_{Z}-\gamma\right)$ são os autovalores da jacobiana associada ao sistema (2.2) linearizado em torno do equilíbrio trivial. Por outro lado, $E_{1}=(\bar{V}, \bar{Y}, \bar{Z})$, dado pela equação (2.3), será L.A.E se

$$
\mu_{V}<1 \text { e } \mu_{Z}>\gamma
$$

condições decorrentes da aplicação do critério de Routh-Hurwitz (não mostrado aqui) aos coeficientes do polinômio característico $p(\lambda)=\lambda^{3}+a_{2} \lambda^{2}+a_{1} \lambda+a_{0}$, com

$$
\begin{aligned}
& a_{2}=\mu_{Y}+(\alpha+\beta) \bar{V}+\mu_{Z}-\gamma, \\
& a_{1}=\left(\mu_{Y}+(\alpha+\beta) \bar{V}\right)\left(\mu_{Z}-\gamma\right)+\bar{V}(\alpha+\beta) \bar{Y}+\eta \bar{V} \beta \bar{Y}, \\
& a_{0}=\bar{V}(\alpha+\beta) \bar{Y}\left(\mu_{Z}-\gamma\right)+\eta \bar{V} \mu_{Y} \beta \bar{Y}
\end{aligned}
$$

associado a jacobiana do sistema (2.2) linealizado em torno do ponto de equilíbrio não-trivial.

Quando $\mu_{Z}<\gamma$, os dois pontos de equilíbrio $E_{0}$ e $E_{1}$ são instáveis. Nesta situação, as células efetoras $Z$ crescem indefinidamente.

A seguir, tem-se ilustrado o comportamento típico das soluções do sistema (2.2). A Figura 1 (a) representa uma situação em que, após o surgimento de partículas virais na corrente sanguínea, a concentração de células T-CD4 ${ }^{+}$inicialmente decai de seu valor normal (representado pelo número 1, no eixo vertical) e depois retorna, enquanto isso, ocorre um aumento da concentração de células efetoras (resposta imunológica). Ao final, as concentrações de vírus, células T-CD4 ${ }^{+}$e efetoras fixamse, respectivamente, nos valores 0,1 e 0 , dados pelo ponto de equilíbrio trivial $E_{0}$. Em (b), temos uma situação em que inicialmente a concentração viral aumenta e após atingir um pico, representando uma alta concentração viral, decresce e fixa-se em uma baixa concentração, correspondente ao valor dado pelo ponto de equilíbrio não-trivial $E_{1}$. Na Figura 1 (c), teríamos a eliminação das partículas virais e recuperação do número de células $\mathrm{T}^{-\mathrm{CD}} 4^{+}$a níveis normais, no entanto, uma vez iniciada a resposta imunológica, a concentração de células efetoras segue crescendo indefinidamente. 


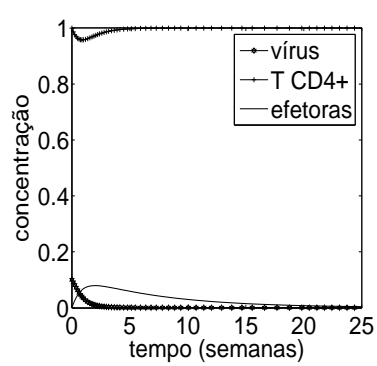

(a) $\mu_{V}>1$ e $\mu_{Z}>\gamma$

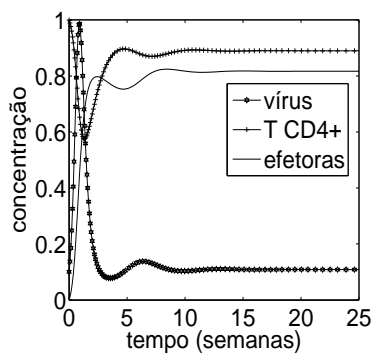

(b) $\mu_{V}<1$ e $\mu_{Z}>\gamma$

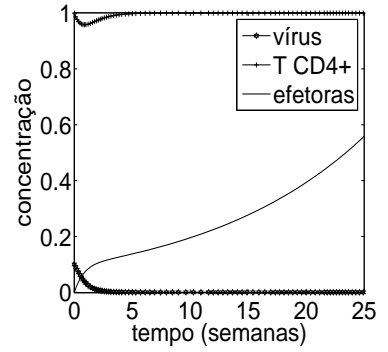

(c) $\mu_{Z}<\gamma$

Figura 1: Comportamento típico das soluções de (2.2). Parâmetros fixos utilizados nestas simulações são: $\eta=0,6 ; \alpha=0,001 ; \beta=0,17 ; \mu_{Y}=0,15 ; \mu_{Z}=0,25$. Parâmetros variáveis são $\mu_{V}$ e $\gamma$, que assumem, respectivamente: (a) 1,1 e 0,23; (b) 0, 4 e 0, 23; e (c) 1, 1 e 0, 26. Valores iniciais utilizados são: $V(0)=0,1 ; Y(0)=1$ e $Z(0)=0$.

\section{Modelo Incluindo Diferenciação Dependente de Vírus}

No modelo anterior, vimos que um comportamento atípico da resposta do SI ao vírus está diretamente relacionado à taxa de diferenciação das células ativadas. Quando $\mu_{Z}<\gamma$, a população de células efetoras cresce indefinidamente, conforme mostrado na Figura 1 (c). O estímulo para diferenciação ou clonagem de células ativadas depende de citocinas liberadas por células como macrófagos. Como a concentração de macrófagos que secretam citocinas depende de concentração de antígenos circulantes, permite-se que a diferenciação de células ativadas seja proporcional também à concentração de vírus. Um outro fator biológico implícito é o fato de que, quando um antígeno é eliminado, as células que foram ativadas para combatê-lo são programadas para morrer, por um processo chamado apoptose. Salvo células de memória, que recebem estímulos para viver e assim proteger o organismo por mais tempo, as quais não foram consideradas no modelo.

O próximo modelo reproduz o processo de diferenciação dependente de vírus, que mimetiza a presença do vírus para fornecer estímulos para diferenciação. Matematicamente, isto é dado pela troca do termo $\gamma$, do modelo anterior, por $\gamma V$. Com isso, temos

$$
\begin{aligned}
& \frac{d V}{d t}=Y V-\mu_{V} V-\eta V Z \\
& \frac{d Y}{d t}=\mu_{Y}-\mu_{Y} Y-\alpha V Y-\beta V Y \\
& \frac{d Z}{d t}=\beta V Y-\mu_{Z} Z+\gamma V Z .
\end{aligned}
$$

Para encontrarmos os pontos de equilíbrio, procedemos como no caso anterior. 
O equilíbrio trivial será $E_{0}=(0,1,0)$ e o não-trivial, $E_{1}=(\bar{V}, \bar{Y}, \bar{Z})$, com

$$
\bar{Y}=\frac{\mu_{Y}}{\mu_{Y}+(\alpha+\beta) \bar{V}} \quad \text { e } \quad \bar{Z}=\frac{\beta \overline{V Y}}{\mu_{Z}-\gamma \bar{V}}
$$

sendo $\bar{V}$ solução positiva da equação do segundo grau

$$
\frac{\gamma(\alpha+\beta)}{\mu_{Y}} \bar{V}^{2}-\left[\left(1-\mu_{V}\right) \frac{\gamma}{\mu_{V}}+(\alpha+\beta) \frac{\mu_{Z}}{\mu_{Y}}+\frac{\eta \beta}{\mu_{V}}\right] \bar{V}+\left(1-\mu_{V}\right) \frac{\mu_{Z}}{\mu_{V}}=0 .
$$

Devido à imposição de que as variáveis $V, Y$ e $Z$ devem ser positivas, dado que representam populações, verificamos que a única solução biologicamente viável será

$$
\bar{V}=\left[\left(1-\mu_{V}\right) \frac{\gamma}{\mu_{V}}+(\alpha+\beta) \frac{\mu_{Z}}{\mu_{Y}}+\frac{\eta \beta}{\mu_{V}}-\sqrt{\Delta}\right] \frac{\mu_{Y}}{2 \gamma(\alpha+\beta)},
$$

sendo $\Delta$ o discriminante da equação (3.3). Portanto, temos apenas um ponto de equilíbrio não-trivial. Quanto à estabilidade, os equilíbrios trivial e o único nãotrivial são:

$$
\begin{aligned}
& \text { (a) } \mu_{V}>1 \Longrightarrow E_{0} \text { é } \quad \text { L.A.E. } \\
& \text { (b) } \mu_{V}<1 \Longrightarrow E_{1} \text { é L.A.E. }
\end{aligned}
$$

A condição $(a)$ segue da imposição de que a parte real dos autovalores da matriz jacobiana do sistema (3.1), linearizado em torno do ponto $E_{0}$, seja negativa. Nesse modelo, eles são dados por $\lambda_{1}=1-\mu_{V}, \lambda_{2}=-\mu_{Y}$ e $\lambda_{3}=-\mu_{Z}$. Aplicando o critério de Routh-Hurwitz (não mostrado aqui) aos coeficiente do polinômio característico associado ao ponto de equilíbrio não-trivial $p(\lambda)=\lambda^{3}+a_{2} \lambda^{2}+a_{1} \lambda+a_{0}$,

$$
\begin{aligned}
& a_{2}=\mu_{Y}+(\alpha+\beta) \bar{V}+\mu_{Z}-\gamma \bar{V}, \\
& a_{1}=\left(\mu_{Y}+(\alpha+\beta) \bar{V}\right)\left(\mu_{Z}-\gamma \bar{V}\right)+\bar{V}(\alpha+\beta) \bar{Y}+\eta \bar{V}(\beta \bar{Y}+\gamma \bar{Z}), \\
& a_{0}=\bar{V}(\alpha+\beta) \bar{Y}\left(\mu_{Z}-\gamma \bar{V}\right)+\eta \bar{V} \mu_{Y}(\beta \bar{Y}+\gamma \bar{Z})+\eta \bar{V}(\alpha+\beta) \bar{V} \gamma \bar{Z},
\end{aligned}
$$

obtemos a condição $(b)$.

O comportamento das soluções deste modelo é semelhante ao das Figuras 1 (a) e (b), excluindo-se o tipo de comportamento da Figura 1 (c).

\section{Dinâmica de HIV de Longo Termo}

Inicialmente, através dos dois modelos anteriores, abordamos de maneira simplificada o início da infecção pelo HIV, até o ponto em que o vírus se estabelece na corrente sanguínea. Nesta seção, propomos um modelo para estágios mais avançados.

Após a fase aguda de infecção, o número de partículas virais é reduzido e por alguns anos o SI permanece competente para lidar com a maioria das infecções por microorganismos oportunistas. No entanto, mesmo com uma baixa carga viral, 
o número de células $\mathrm{T}_{-} \mathrm{CD} 4^{+}$continua a diminuir, deixando o SI cada vez mais vulnerável.

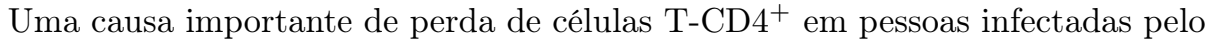
HIV diz respeito aos efeitos citopáticos diretos da infecção dessas células pelo HIV [1]. Esta redução do número de T-CD4 ${ }^{+}$é um fator que compromete o SI. No entanto, mesmo antes da depleção significativa de células T-CD4 ${ }^{+}$, defeitos no SI de indivíduos infectados por HIV já são detectáveis [1]. Assim, é razoável supor que a persistente presença do vírus produz um enfraquecimento do SI, pelo continuado estímulo. Representamos por $E$ o enfraquecimento do SI. Sabemos da literatura que o número de partículas virais pode tornar a crescer quando o SI encontra-se debilitado. Sendo assim, supomos uma diminuição na resposta humoral à população viral proporcional a $E$, sendo a constante de proporcionalidade denotada por $\tilde{\lambda}$. Com isso, temos o seguinte modelo:

$$
\begin{aligned}
& \frac{d V}{d t}=Y V-\mu_{V} V-\eta V Z+\widetilde{\lambda} E V \\
& \frac{d Y}{d t}=\mu_{Y}-\mu_{Y} Y-\alpha V Y-\beta V Y-E \\
& \frac{d Z}{d t}=\beta V Y-\mu_{Z} Z+\gamma Z,
\end{aligned}
$$

sendo

$$
E=\varphi \frac{\widetilde{Z}^{n}}{\widetilde{Z}^{n}+F^{n}} \quad \text { e } \quad \widetilde{Z}=\int_{0}^{t} Z(s) d s .
$$

Escolhemos $E$ desta maneira por estar de acordo com as seguintes considerações: (a) inicialmente, o SI permanece competente para lidar com a maioria das infecções por microorganismos oportunistas. Portanto, se uma expressão puder descrever o processo de enfraquecimento, deve ser tal que no início não influencie muito; (b) como a presença do vírus estimula a ativação de células para atacá-lo e, ao mesmo tempo, o SI tenta responder à infecção, ele fornece células para o vírus infectar, além do fato de que a reação e os produtos desta constante batalha entre vírus e SI pode aumentar a citotoxidade celular, de alguma forma $E$ deve depender de $Z$, pois a resposta do SI é dada por $Z$; e (c) quando o número de células T-CD4 ${ }^{+}$encontra-se abaixo de 200 células $/ \mathrm{mm}^{3}$ tem-se um alto risco para doenças oportunistas e isto ocorre (considerando apenas o curso natural da doença) em média por volta de uns oito a dez anos. Desta forma, o enfraquecimento deve ser algo resultante de um esforço cumulativo ao longo dos anos, o qual representamos por $\widetilde{Z}$.

Para que (4.2) esteja de acordo com os ítens (a), (b) e (c), poderíamos escolher vários valores para $n$, mas decidimos trabalhar com $n=2$. Como veremos através das simulações numéricas, parece uma escolha satisfatória. Interpretamos o parâmetro $F$ que aparece em (4.2) como sendo o quanto o SI consegue resistir a infecção e o parâmetro $\varphi$ como uma taxa ponderando o enfraquecimento.

A seguir, delineamos como abordamos matematicamente o modelo (4.1). Introduzindo a mudança de variáveis $\lambda=\widetilde{\lambda} \varphi$ e $\varphi=\theta \mu_{Y}$, (4.1) assume a forma

$$
\frac{d V}{d t}=Y V-\mu_{V} V-\eta V Z+\lambda \frac{\widetilde{Z}^{2}}{\widetilde{Z}^{2}+F^{2}} V
$$




$$
\begin{aligned}
& \frac{d Y}{d t}=\mu_{Y}\left(1-\frac{\widetilde{Z}^{2}}{\widetilde{Z}^{2}+F^{2}} \theta\right)-\mu_{Y} Y-\alpha V Y-\beta V Y \\
& \frac{d Z}{d t}=\beta V Y-\mu_{Z} Z+\gamma Z .
\end{aligned}
$$

Para $F$ suficientemente grande, o termo $\widetilde{Z}^{2} /\left(\widetilde{Z}^{2}+F^{2}\right)$ tem pouca influência na dinâmica de (4.3). Portanto, desprezando este termo, a dinâmica da fase inicial de infecção é semelhante à de (2.2). No caso em que o vírus consegue se estabelecer na corrente sanguínea, $\mu_{V}<1$ e $\mu_{Z}>\gamma$, como a variável $Z$ é positiva, a integral $\widetilde{Z}$ dada em (4.2) é divergente. Além disso, $\lim _{t \rightarrow \infty} \widetilde{Z}^{n} /\left(\widetilde{Z}^{n}+F^{n}\right)=1$. Logo, para procurar os pontos de equilíbrio do sistema (4.3), supondo que o vírus se estabeleça na corrente sanguínea, procuramos as soluções de

$$
\begin{aligned}
& \overline{Y V}-\mu_{V} \bar{V}-\eta \overline{V Z}+\lambda \bar{V}=0 \\
& \mu_{Y}(1-\theta)-\mu_{Y} \bar{Y}-\alpha \overline{V Y}-\beta \overline{V Y}=0 \\
& \beta \overline{V Y}-\mu_{Z} \bar{Z}+\gamma \bar{Z}=0 .
\end{aligned}
$$

Para a existência de um equilíbrio trivial $(\bar{V}=0)$ devemos ter $0<\theta<1$, visto que este equilíbrio seria $P_{0}=(\bar{V}, \bar{Y}, \bar{Z})=(0,1-\theta, 0)$. Se $\bar{V} \neq 0$, temos a solução não-trivial, $P_{1}$, dada por

$$
\left(\frac{\mu_{Y}\left(\mu_{Z}-\gamma\right)\left(1-\theta-U_{V}\right)}{U_{V}\left(\mu_{Z}-\gamma\right)(\alpha+\beta)+\eta \beta \mu_{Y}(1-\theta)}, \frac{\mu_{Y}(1-\theta)}{\mu_{Y}+(\alpha+\beta) \bar{V}}, \frac{\beta \overline{V Y}}{\mu_{Z}-\gamma}\right),
$$

sendo $U_{V}=\mu_{V}-\lambda$. Impondo que $\bar{Y}$ e $\bar{Z}$ sejam positivos, devemos ter $0<\theta<1$ e $\mu_{Z}>\gamma$. Para estudar quando $\bar{V}$ é positivo deveríamos analisar os casos $U_{V}>0$ e $U_{V}<0$. Mas, por simplicidade, consideremos apenas o caso $U_{V}<0$. Nesta situação, $\bar{V}$ será positivo se

$$
\left|U_{V}\right|<\frac{\eta \beta \mu_{Y}(1-\theta)}{\left(\mu_{Z}-\gamma\right)(\alpha+\beta)}
$$

desigualdade obtida impondo que o denominador da expressão dada para $\bar{V}$, em (4.4), seja positivo. A Figura 2 ilustra o comportamento da solução para parâmetros satisfazendo (4.5) e a semelhança, durante a fase inicial, entre os resultados obtidos com os modelos (4.1) e (2.2). Os gráficos desta figura são para quando as restrições $\mu_{V}<1$ e $\mu_{Z}>\gamma$ também são safisfeitas, ou seja, o vírus se estabelece na corrente sanguínea. Fixando $\mu_{V}>1$ e $\mu_{Z}>\gamma$, mantendo o mesmo valor dos demais parâmetros fixados para a figura anterior, para os três valores de $F$, o sistema se estabiliza em $E_{0}=(0,1,0)$.

Na Figura 2, o valor 1 para a concentração representa o valor de homeostase das células T-CD $4^{+}$, em média, 1000 células $/ \mathrm{mm}^{3}$. Sendo assim, podemos pensar o eixo vertical como estando na escala 1:1000. Note que, na fase inicial, os gráficos gerados pelos dois modelos são semelhantes e a concentração de células T-CD4 ${ }^{+}$está um pouco abaixo do valor de homeostase. O período em que o SI consegue responder a infecção, mantendo o número de células T-CD4 ${ }^{+}$, está diretamente relacionado com 

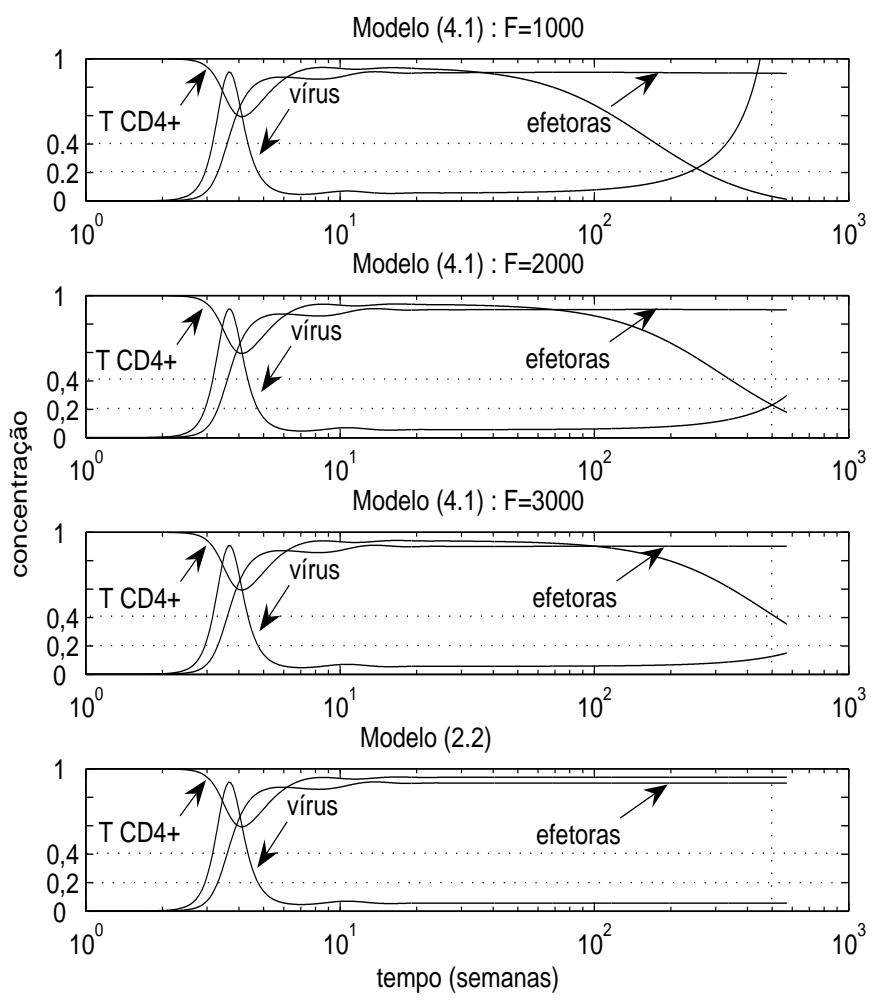

Figura 2: Comportamento típico das soluções de (4.1). Durante a fase inicial de infecção, os modelos (4.1) e (2.2) se comportam de maneira semelhante. A fase assintomática aumenta com o valor de $F$, isto é percebido pela elevação da curva que representa as células T-CD4 ${ }^{+}$.

o valor do parâmetro $F$. No primeiro gráfico, $F=1000$, observa-se que, ao longo de aproximadamente 10 anos (linha vertical pontilhada), a concentração de células $\mathrm{T}-\mathrm{CD} 4^{+}$ficou bem abaixo de 200 células $/ \mathrm{mm}^{3}$; no segundo, aproximadamente 200 células $/ \mathrm{mm}^{3}$; e no terceiro, por volta de 400 células $/ \mathrm{mm}^{3}$. Podemos observar nestes gráficos que quanto maior o valor de $F$, por mais tempo o SI mantém o número de células T-CD4 ${ }^{+}$elevado.

\section{O Uso de Medicamentos}

Até o momento, dispõe-se das seguintes classes de drogas ARV's para o tratamento do HIV: inibidores da transcriptase reversa análogos de nucleosídeos, inibidores da transcriptase reversa não análogos de nucleosídeos, inibidores da protease, inibidores da integrase, antagonistas de CCR5 e inibidores de fusão [7]. 
Com a disponibilidade de diferentes classes de ARV's e o uso de coquetéis antiHIV (associação desses medicamentos) foi possível reduzir a morbidade e mortalidade de indivíduos soropositivos [5].

A modelagem de um tratamento pode ser feita representando a eficiência de ARV's como parâmetros que assumem valores entre 0 e 1 [9] [11], por meio de equações diferenciais impulsivas [12], ou ainda modelando a dinâmica de drogas ARV's [13]. Neste trabalho, para ilustrar como o uso de medicamentos pode afetar os resultados aqui obtidos, utilizamos a primeira maneira.

Incluímos a eficiência de um tratamento em (4.1) trocando o termo (4.2) por

$$
E=\varphi \frac{[(1-D) \widetilde{Z}]^{n}}{[(1-D) \widetilde{Z}]^{n}+F^{n}}, \quad \text { com } \quad D \in[0,1] \quad \text { e } \quad \widetilde{Z}=\int_{0}^{t} Z(s) d s,
$$

onde $D$ representa a eficiência de um coquetel anti-HIV.

Ao considerarmos a variável $\widetilde{Z}$ multiplicada por $(1-D)$, implicitamente supomos que a eficiência de um coquetel pode reduzir o esforço necessário para conter a infecção. O valor $D=1$ corresponde a $100 \%$ de eficiência, caso em que $E=0$, e, a dinâmica do modelo reduz-se a de (2.2). Se $D=0$, tem-se o modelo sem tratamento. Supondo $0<D<1$, podemos reescrever (5.1) como

$$
E=\varphi \frac{\widetilde{Z}^{n}}{\widetilde{Z}^{n}+\left(\frac{F}{1-D}\right)^{n}}, \quad \text { com } \quad D \in(0,1) \quad \text { e } \quad \widetilde{Z}=\int_{0}^{t} Z(s) d s
$$

Segundo esta expressão, quanto mais eficiente for o medicamento por mais tempo o SI pode resistir a infecção e menos o enfraquecimento será sentido (para ver isto, basta pensar em $D$ próximo de 1, e lembrar das interpretações de $E$ e $F$ ). Em outras palavras, a consideração do uso de medicamentos pode influenciar os resultados do modelo alterando o efeito de $F$, como se simplesmente trocássemos o valor deste parâmetro, no modelo sem tratamento, por outro maior e equivalente a $F_{D}=F /(1-D)$.

\section{Conclusões}

Com os dois primeiros modelos, abordamos a fase inicial da infecção pelo HIV, e, observamos que se a diferenciação não depender da sinalização do antígeno, as células efetoras podem crescer indefinidamente (Figura 1(c)).

Para os três modelos, a condição $\mu_{V}>1$ é crucial para garantir a estabilidade do ponto de equilíbrio representativo da situação em que não há vírus na corrente sanguínea. Ao reescrevermos esta condição em termos dos parâmetros dimensionais, encontramos um limiar $\alpha_{0}=\mu_{V} / a(1-f) Y_{h}$, que não depende da ação de anticorpos mas do efeito das células T-CD $8^{+}$. O vírus é eliminado para valores de $\alpha$, taxa de infecção das células pelo vírus, abaixo deste limiar e se estabelece para valores maiores. Desta forma, quanto maior $\alpha_{0}$ mais eficiente o vírus deve ser para conseguir propagar a infecção.

Analisando $\alpha_{0}$, que depende de $\mu_{V}, a, f$ e $Y_{h}$, podemos observar que se o número de partículas virais produzidas por célula infectada (parâmetro a) ou o valor 
de homeostase das células T-CD4 ${ }^{+}$(parâmetro $Y_{h}$ ) forem elevados, o limiar tornase menor e, portanto, maior a dificuldade para eliminar o vírus. Por outro lado, o aumento do valor de $\mu_{V}$, que corresponde a inativação viral, eleva o limiar. A intervenção por meio do uso de anti-retrovirais, como os inibidores de transcriptase reversa que, incorporando-se à cadeia de DNA que o virus cria, tornam-na defeituosa, pode ser considerada como uma forma de aumentar o valor de $\mu_{V}$ e, consequentemente, do limiar. Ainda analisando $\alpha_{0}$, observamos que outra possível intervenção para eliminar o vírus seria pensar em estratégias que melhorem o efeito das células $\mathrm{T}_{-} \mathrm{CD} 8^{+}$(aproximar f de 1 ).

Através da introdução do termo de exaustão cumulativa, com o terceiro modelo, além da fase inicial, foi possível reproduzir a diminuição do número de células T$\mathrm{CD}^{+}$ao longo dos anos e o crescimento da população viral (Figura 2).

Esperamos que este trabalho possa nortear ou, como em nosso caso, servir de base para pesquisas posteriores. Uma vez que traz a modelagem de expressões ainda não vistas em outros modelos, tais como: enfraquecimento do SI, representada pelo termo de exaustão cumulativa $(E)$; resistência do SI $(\mathrm{F})$; e esforço cumulativo $(\widetilde{Z})$.

As expressões comentadas servem, por exemplo, como uma forma alternativa de abordar a importante questão, ainda polêmica, "Qual o momento ideal para iniciar um tratamento com ARV's?". Subsídios para a resposta talvez sejam encontrados em outras questões como: "Qual o resultado esperado ao iniciar o tratamento em um determinado momento em que o SI encontra-se com um certo grau de enfraquecimento?", "Quando a resistência do SI pode ser considerada suficiente para resistir a infecção sem o uso de medicamentos?". Claramente, estas também são difíceis de serem respondidas, mas os modelos aqui apresentados trazem uma primeira formulação matemática dos termos necessários para abordá-las.

Um próximo desafio seria tentar quantificar algum dos parâmetros introduzidos neste trabalho, confrontando com dados de pacientes. Neste estágio, possivelmente mais características biológicas serão incluídas no modelo. Mas, isto é assunto para outro trabalho.

\begin{abstract}
The Human Immunodeficiency Virus (HIV) infecting T-CD4 ${ }^{+}$cells can severely compromise the functioning Immune System, resulting in AIDS. We have constructed and analyzed three mathematical models for this process of infection. It was possible to find a threshold for the permanence of virus in the blood and highlight the need for apoptosis of activated cells after the elimination of antigen. The introduction of a exhausting term (result of the constant attempt of the immune system to respond to HIV infection) also reproduced the decrease in the number of $\mathrm{T}-\mathrm{CD} 4^{+}$cells over the years.
\end{abstract}

\title{
Referências
}

[1] A.K. Abbas, "Imunologia Celular e Molecular", Elsevier, Rio de Janeiro, 2005.

[2] F. Barré-Sinoussi et al. Isolation of a T-lymphotropic retrovírus from a patient at risk for acquired immune deficiency syndrome (AIDS). Science, 220 (1983), 868-871. 
[3] S. Barrozo, H.M. Yang, Mecanismos da iteração antígeno-anticorpo em uma resposta primária célula T-mediada, Tend. Mat. Apl. Comput., 7, No.1 (2006) $43-52$.

[4] S. Barrozo, H.M. Yang, Desenvolvimento de um modelo para resposta imunológica primária célula-mediada, Tend. Mat. Apl. Comput., 7, No.1 (2006) 31-41.

[5] P.F. Bonolo et al. Adesão à terapia anti-retroviral (HIV/aids): fatores associados e medidas da adesão, Epidemiol. Serv. Saúde 16 (2007), 251-259.

[6] R.C. Gallo, P.S. Sarin, E.P. Gelmann et al. Isolation of human T-cell leukemia virus in acquired immunodeficiency syndrome (Aids). Science, 220 (1983), 865867.

[7] A. Hughes, T. Barber, M. Nelson, New treatment options for HIV salvage patients: An overview of second generation Pis, NNRTIs, integrase innibitors and CCR5 antagonists. J. Infect, London, 57 (2008), 1-10.

[8] C.A. Janeway Jr., How the immune system reconizes invaders. Scientific American, 269 (1993), 73-79.

[9] H. Kim, A.S. Perelson, Viral and Latent Reservoir Persistence in HIV-1-Infected Patients on Therapy, PLoS Cumput. Biol. 2 (2006), 1232-1247.

[10] M. Oprea, A.S. Perelson, Exploring the mechanisms of primary antibory responses to T-cell dependent antigens, J. Theor. Biol., 181 (1996), 215-236.

[11] A.S. Perelson, P.W. Nelson, Mathematical analysis of HIV-I dynamics in vivo, Society for Industrial and Applied Mathematics, 41 (1999), 3-44

[12] R.J. Smith, B.D. Aggarwala, Can the viral reservoir of latently infected CD4 ${ }^{+}$ T cells be eradicated with antiretroviral HIV drugs?, J. Math. Biol., 59 (2009), $697-715$.

[13] R.J. Smith, Explicitly accounting for antiretroviral drug uptake in theoretical HIV models predicts long-term failure of protease-onli therapy Journal of Theoretical Biology, 251 (2008), 227-237.

[14] L. Sompayrac, "How Pathogenic Vírus Work", Jones and Bartlett Publishers, Massachusetts, 2002. 